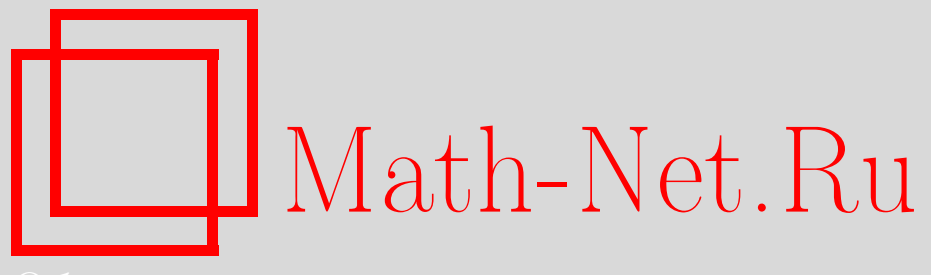

А. А. Дороговцев, В. В. Бакунин, Случайные отображения и обобщенные аддитивные функционалы от винеровского процесса, Теория вероятн. и ее примен., 2003, том 48, выпуск 1, 43-61

DOI: https://doi.org/10.4213/tvp300

Использование Общероссийского математического портала MathNet.Ru подразумевает, что вы прочитали и согласны с пользовательским соглашением

http://www . mathnet.ru/rus/agreement

Параметры загрузки:

IP : 3.85 .73 .92

26 апреля 2023 г., 03:22:09

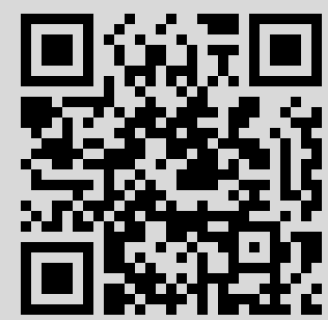


(c) 2003 г.

ДОРОГОВЦЕВ А.А.*, БАКУН В.В.**

\section{СЛУЧАЙНЫЕ ОТОБРАЖЕНИЯ И ОБОБЩЕННЫЕ АДДИТИВНЫЕ ФУНКЦИОНАЛЫ ОТ ВИНЕРОВСКОГО ПРОЩЕССА}

В статье введено определение обобщенного аддитивного однородного функционала от винеровского процесса. Показано, что обобщенный функционал однозначно задается своей характеристикой, при этом роль порождающих функций играют функции из пространства Шварца $S^{*}$ медленно растущих обобшенных функций.

Ключевые слова и фразы: обобщенные винеровские функционалы, кратные стохастические интегралы, аддитивные функционалы.

1. Введение. Пусть $(\Omega, \mathscr{F}, \mathbf{P})$-- вероятностное пространство, $\{w(t) ; t \in[0 ; 1]\}-\mathbf{R}$-значный винеровский процесс на $[0 ; 1]$, заданный на $(\Omega, \mathscr{F}, \mathbf{P})$ и стартующий из нуля $(w(0)=0)$. Через $\widetilde{w}$ обозначим процесс Маркова в $\mathbf{R}$ с винеровской переходной вероятностью на $[0 ; 1]$ (в терминологии [1] $\widetilde{w}-$ марковский процесс, $w-$ марковская случайная функция). Цель настоящей работы - исследование аддитивных функционалов от $\widetilde{w}$ вида

$$
\varphi_{t}^{s}=\int_{s}^{t} \varkappa(\widetilde{w}(r)) d r
$$

Здесь $\varkappa$ - обобщенная функция из пространства $S^{*}$ функций медленного роста на $\mathbf{R}$. В том случае, когда $\varkappa$ есть $\delta$-функция или, более общо, конечная мера, (1.1) превращается в локальное время для винеровского процесса. Рассмотрение функционалов типа (1.1) с произвольным $\varkappa$ требует определения соответствующего интеграла. В работах [2]-[5], [12] такие интегралы рассматривались как обобщенные случайные величины, определяемые наборами своих ядер из разложения Ито-Винера. При этом возникала необходимость в некоторой регуляризации, что приводило к потере свойства аддитивности. В этой статье мы вводим такое определение обобщенного аддитивного функционала от $\tilde{w}$,

\footnotetext{
${ }^{*}$ Институт математики НАН Украины, ул. Терещенковская, 3, 01601 Киев, Украина; e-mail: adoro@imath.kiev.ua

${ }^{* *}$ Киевский политехнический институт, просп. Победы, 37, 252056 Киев, Украина.
} 
которое включает в себя интегралы типа (1.1), исследуем (1.1) для различных обобщенных функций $\varkappa$, приводим теорему, позволяющую восстановить $\varkappa$ по характеристике обобщенного аддитивного функционала.

2. Обобщенные случайные отображения, связанные с процессом $\widetilde{w}$. Обозначим $\mathscr{F}^{s, t}=\sigma(w(\tau)-w(s), \tau \in[s ; t])$ при $0 \leqslant s \leqslant t \leqslant 1$. Известно, что произвольная случайная величина $\alpha \in L_{2}(\Omega, \mathscr{F}, \mathbf{P})$, будучи $\mathscr{F}^{s, t}$-измеримой, однозначно представляется в виде

$$
\alpha=\sum_{k=0}^{\infty} I_{k}^{s, t}\left(a_{k}\right)
$$

где $I_{k}^{s, t}\left(a_{k}\right)-k$-кратный интеграл Ито от симметричного ядра $a_{k} \in$ $L_{2}\left([s ; t]^{k}\right), k \geqslant 1, \mathbf{и}$

$$
\mathbf{E} \alpha^{2}=\sum_{k=0}^{\infty} k !\left\|a_{k}\right\|_{k}^{2} .
$$

Здесь $\left\|a_{k}\right\|_{k}$ - норма в $L_{2}\left([s ; t]^{k}\right), k \geqslant 1, a_{0}=\mathbf{E} \alpha \in \mathbf{R},\left\|a_{0}\right\|_{0}=\left|a_{0}\right|$. Обозначим через $W_{-p}^{s, t}$ для $p>0$ пополнение $L_{2}\left(\Omega, \mathscr{F}^{s, t}, \mathbf{P}\right)$ в норме

$$
\|\alpha\|_{-p}^{a}:=\sum_{k=0}^{\infty} k !(k+1)^{-p}\left\|a_{k}\right\|_{k}^{2} .
$$

Пространство $W_{-p}^{s, t}$ можно рассматривать как совокупность обобщенных случайных величин, имеющих порядок $p$ (см. [5], [6]).

Пусть $B$ - вещественное сепарабельное банахово пространство с нормой $\|\cdot\|_{B}$.

О п р е д е л н и е 2.1. Обобщенным случайным отображением порядка $p \geqslant 0$ пространства $B$ в $\mathbf{R}$ называется измеримая функция $G: B \rightarrow W_{-p}^{0,1}$.

Отметим, что случайному отображению соответствует набор неслучайных измеримых функций-ядер $a_{k}: B \rightarrow L_{2}\left([0 ; 1]^{k}\right)$, с помощью которого $G$ записывается формальным рядом

$$
G(u)=\sum_{k=0}^{\infty} I_{k}^{0,1}\left(a_{k}(u)\right) .
$$

Даже в том случае, когда порядок $p=0$, т.е. когда значения $G$ - это обычные случайные величины из $L_{2}\left(\Omega, \mathscr{F}^{0,1}, \mathbf{P}\right), G$ может не быть заиндексированным $\omega \in \Omega$ набором «хороших» детерминированных функций (см. [7], [8]). Поэтому действие $G$ на случайные элементы в $B$ нуждается в дополнительном определении. В случае $p=0$ такое определение было введено в [7], [9]. Здесь мы приводим модификацию на случай произвольного $p>0$. Пусть $\zeta-$ случайный элемент в $B$, являющийся $\mathscr{F}^{0,1}$-измеримым и такой, что

$$
\forall k \geqslant 0: \mathbf{E}\left\|a_{k}(\zeta)\right\|_{k}^{2}<+\infty
$$


Тогда для каждого $k \geqslant 0$ можно рассмотреть $L_{2}\left([0 ; 1]^{k}\right)$-значное разложение Ито-Винера:

$$
a_{k}(\zeta)=\sum_{j=0}^{\infty} I_{j}^{0,1}\left(a_{k j}\right)
$$

При каждом $j$ здесь $a_{k j}$ является симметричной $L_{2}\left([0 ; 1]^{k}\right)$-значной функцией на $[0 ; 1]^{j}$, интегрируемой с квадратом. Поэтому, после симметризации по всем переменным из $[0 ; 1]$, из $a_{k j}$ получается ядро $\Lambda a_{k j}$, являющееся элементом пространства $L_{2}\left([0 ; 1]^{k+j}\right)$.

О п р е д е л е н и 2.2. Случайный элемент $\zeta$ из $B$ входит в область определения случайного отображения $G$, если формальный ряд

$$
G(\zeta)=\sum_{n=0}^{\infty}\left(\sum_{j=0}^{n} I^{0,1}\left(\Lambda a_{n-j, j}\right)\right)
$$

является элементом $W_{-p}^{0,1}$; сумма $(2.1)$ называется значением $G$ на $\zeta$.

Следующий пример показывает, что в некоторых простых ситуациях определение 1.2 приводит к обычной суперпозиции.

Лемма 2.1. Пусть $F: C([0 ; 1]) \rightarrow \mathbf{R}-$ измеримая функиия такая, чmo

$$
\forall u \in C([0 ; 1]), \forall t \in[0 ; 1]: \quad \mathbf{E} F(W(u, t))^{2}<+\infty,
$$

где $W(u, t)(r)=u(r \wedge t)+w(r \vee t)-w(t), r \in[0 ; 1]$.

Тогда для всякого $t \in[0 ; 1]$ соответствие

$$
C([0 ; t]) \ni u \mapsto F(W(u, t))=G_{t}(u)
$$

является случайным отображением, принимающим значения в $L_{2}^{t, 1}$. $\Pi p и$ этом для всех $s \in[0 ; t), v \in C([0 ; s])$ случайный элемент $W(v, s, t)$ $($ где $W(v, s, t)(r)=v(r \wedge s)+w(r \wedge t)-w(r \wedge s))$ входит в область определения $G_{t} u$

$$
G_{t}(W(v, s, t))=G_{s}(v)
$$

Д о к а з а т е л ь с т в о. Проверим измеримость $G_{t}$ как отображения, действующего из $C([0 ; t])$ в $L_{2}^{t, 1}$. Для этого заметим, что $L_{2}^{t, 1}-$ сепарабельное гильбертово пространство, и, следовательно, достаточно убедиться в том, что для всякой случайной величины $\alpha \in L_{2}^{t, 1}$ отображение

$$
C_{t}: C([0 ; t]) \ni u \longmapsto \mathbf{E} F(W(u, t)) \alpha
$$

измеримо. Последнее следует из стандартных аргументов теории меры. Второе утверждение леммы следует из приведенного в [8] свойства суперпозиции независимых случайных $L_{2}$-значных отображений.

Следующая лемма описывает свойства суперпозиции обобщенных случайных отображений. 
Лемма 2.2. Пусть $G$ - случайное отображение пространства $C([0 ; s])$ со значениями в $W_{-p}^{s, t}$ для некоторых $p>0,0<s<t \leqslant 1$, такое, что для любого $r \in(0 ; s)$ u любого $v \in C([0 ; r])$

$$
\mathbf{E} \sum_{k=0}^{\infty} k !(k+1)^{-p}\left\|a_{k}(W(v, r, s))\right\|_{k}^{2}<+\infty
$$

где $\left\{a_{k}\right\}-$ коэфФиииенты разложения Ито-Винера для $G$. Тогда при каждом $r \in(0 ; s)$ соответствие

$$
C([0 ; r]) \ni v \mapsto G(W(v, r, s))
$$

определено и является случайным отображением со значениями в $W_{-p-1}^{r, t}$.

Д о к а з а т е л ь с т в о. Рассмотрим при фиксированном $k \geqslant 1$ случайное ядро $a_{k}(W(v, r, s))$. Это интегрируемая с квадратом случайная функция, не зависящая от приращений винеровского процесса на отрезке $[s ; t]$. Поэтому, после разложения $a_{k}(W(v, r, s))=\sum_{j=0}^{\infty} I_{j}^{r, s}\left(a_{k j}\right)$ и соответствующей симметризации $a_{k j} \mapsto \Lambda a_{k j}, j \geqslant 0$, получим ядра, для которых ряд $\sum_{j=0}^{\infty} I_{k+j}^{r, t}\left(\Lambda a_{k j}\right)$ сходится в $L_{2}^{r, t}$ к кратному интегралу Ито от случайного ядра $a_{k}(W(v, r, s))[7],[9]$. При этом

$$
\sum_{j=0}^{\infty}(k+j) !\left\|\Lambda a_{k j}\right\|_{k+j}^{2}=\mathbf{E}\left[I_{k}^{s, t}\left(a_{k}(W(v, r, s))\right)\right]^{2}=k ! \mathbf{E}\left\|a_{k}(W(v, r, s))\right\|_{k}^{2} .
$$

Следовательно,

$$
\begin{aligned}
& \sum_{n=0}^{\infty} n !(n+1)^{-p-1}\left\|\sum_{j=0}^{n} \Lambda a_{n-j, j}\right\|_{n}^{2} \leqslant \sum_{n=0}^{\infty} n !(n+1)^{-p} \sum_{j=0}^{n}\left\|\Lambda a_{n-j, j}\right\|_{n}^{2} \\
& \quad=\sum_{k, j=0}^{\infty}(k+j) !(k+j+1)^{-p}\left\|\Lambda a_{k j}\right\|_{k+j}^{2} \\
& \leqslant \sum_{k=0}^{\infty}(k+1)^{-p} \sum_{j=0}^{\infty}(k+j) !\left\|\Lambda a_{k j}\right\|_{k+j}^{2} \\
& =\sum_{k=0}^{\infty} k !(k+1)^{-p} \mathbf{E}\left\|a_{k}(W(v, r, s))\right\|_{k}^{2}<+\infty
\end{aligned}
$$

Измеримость соответствующих ядер по $v$ сейчас очевидна. Лемма доказана.

Пусть $G$ - случайное отображение банахова пространства $B$, принимающее значения в $W_{-p}^{s, t}, 0<s<t<1, h$ - некоторое положительное число, меньшее $1-t$. Определим сдвиг $G$ вдоль траекторий процесса $w$ на $h$. 
О п р е д е л е н и е 2.3. Сдвиг $\theta_{h} G$ случайного отображения $G-$ это случайное отображение, действующее из $B$ в $W_{-p}^{s+h, t+h}$ по правилу

$$
B \ni u \mapsto \sum_{k=0}^{\infty} I_{k}^{s+h, t+h}\left(a_{k}(u)\right) .
$$

Здесь $\left\{a_{k} ; k \geqslant 0\right\}$ - ядра из разложения Ито-Винера отображения $G$, кратные интегралы $I_{k}^{s+h, t+h}$ берутся по приращениям винеровского процесса $\{w(\tau)-w(s+h), \tau \in[s+h ; t+h]\}$.

$\Pi$ р и м е р 2.1. Пусть $f: \mathbf{R} \rightarrow \mathbf{R}$ - борелевская ограниченная функция. Отображение $G$ устроено следующим образом. Для фиксированных $0<s<t<1$

$$
\mathbf{R} \ni x \mapsto \int_{s}^{t} f(x+w(r)-w(s)) d r=G(x) .
$$

Очевидно, что $G$ - случайное отображение, принимающее значения в $L_{2}^{s, t}$. Сдвиг $G$ на $h \in[0 ; 1-t]$ будет действовать по правилу

$$
\mathbf{R} \ni x \mapsto \int_{s+h}^{t+h} f(x+w(r)-w(s+h)) d r .
$$

Для того чтобы убедиться в этом, достаточно выяснить, как связаны между собой разложения Ито-Винера случайных величин $\varphi(w(\tau)-w(s))$ и $\varphi(w(\tau+h)-w(s+h))$ для произвольной ограниченной борелевской функции $\varphi$. Имеем

$$
\begin{aligned}
& \varphi(w(r)-w(s)) \\
& =\sum_{k=0}^{\infty} \frac{1}{k !} \int_{\mathbf{R}} \varphi(u) H_{k}\left(\frac{u}{\sqrt{r-s}}\right) p_{r-s}(u) d u H_{k}\left(\frac{w(r)-w(s)}{\sqrt{r-s}}\right) \\
& =\sum_{k=0}^{\infty} \frac{1}{k !} \int_{\mathbf{R}} \varphi(u) H_{k}\left(\frac{u}{\sqrt{r-s}}\right) p_{r-s}(u) d u \frac{1}{(\sqrt{r-s})^{k}} I_{k}^{s, r}\left(\mathbb{1}_{[s ; r]}^{\otimes k}\right) .
\end{aligned}
$$

Здесь $H_{k}(x)=(-1)^{k} e^{x^{2} / 2}(d / d x)^{k} e^{-x^{2} / 2}-k$-й многочлен Эрмита со старшим коэффициентом $1, k \geqslant 0, p_{\tau}(u)=(\sqrt{2 \pi \tau})^{-1 / 2} \exp \left(-u^{2} /(2 \tau)\right)$. Поэтому

$$
\begin{aligned}
\sum_{k=0}^{\infty} & \frac{1}{k !} \int_{\mathbf{R}} \varphi(u) H_{k}\left(\frac{u}{\sqrt{r-s}}\right) p_{r-s}(u) d u \frac{1}{(\sqrt{r-s})^{k}} I_{k}^{s+h, r+h}\left(\mathbb{I}_{[s ; r]}^{\otimes k}\right) \\
= & \sum_{k=0}^{\infty} \frac{1}{k !} \int_{\mathbf{R}} \varphi(u) H_{k}\left(\frac{u}{\sqrt{r-s}}\right) p_{r-s}(u) d u \frac{1}{(\sqrt{r-s})^{k}} I_{k}^{s+h, r+h}\left(\mathbb{I}_{[s+h ; r+h]}^{\otimes k}\right) \\
= & \sum_{k=0}^{\infty} \frac{1}{k !} \int_{\mathbf{R}} \varphi(u) H_{k}\left(\frac{u}{\sqrt{(r+h)-(s+h)}}\right) p_{(r+h)-(s+h)}(u) d u \\
& \times H_{k}\left(\frac{w(r+h)-w(s+h)}{\sqrt{(r+h)-(s+h)}}\right)=\varphi(w(r+h)-w(s+h)) .
\end{aligned}
$$


Таким образом, определение 2.3 согласуется с обычным определением сдвига вдоль траекторий процесса $w$ (см. [1]).

3 а м е ч а н и е. Рассуждения примера можно распространить на произвольные квадратично интегрируемые функционалы от $w$.

Сформулируем теперь основное определение данной работы.

О п р е д е л е н е 2.4. Обобщенный однородный аддитивный функционал $G$ порядка $p \geqslant 0$ от процесса $\widetilde{w}$ - это семейство обобщенных случайных отображений $\left\{G_{\varepsilon}^{s, t} ; 0<\varepsilon<s<t \leqslant 1\right\}$, заданных на $\mathbf{R}$ и удовлетворяющих условиям:

1) $G_{\varepsilon}^{s, t}: \mathbf{R} \rightarrow W_{-p}^{s-\varepsilon, t}$;

2) для любых $0<\delta<\varepsilon<s<t \leqslant 1, x \in \mathbf{R}$

$$
\mathbf{E} \sum_{k=0}^{\infty} k !(k+1)^{-p}\left\|a_{k}(x+w(s-\delta)-w(s-\varepsilon), \delta, s, t)\right\|_{k}^{2}<+\infty
$$

где $\left\{a_{k}(u, \delta, s, t) ; k \geqslant 0\right\}$ - ядра из разложения Ито-Винера для $G_{\delta}^{s, t}(u)$;

3) для любых $0<\delta<\varepsilon<s \leqslant 1, x \in \mathbf{R}$

$$
G_{\delta}^{s, t}(x+w(s-\delta)-w(s-\varepsilon))=G_{\varepsilon}^{s, t}(x) ;
$$

4) для любых $0<\varepsilon<s<t<1,0<h<1-t, x \in \mathbf{R}$

$$
\theta_{h} G_{\varepsilon}^{s, t}(x)=G_{\varepsilon}^{s+h, t+h}(x)
$$

5) для любых $0<\varepsilon<s<t<\tau \leqslant 1, x \in \mathbf{R}$

$$
G_{\varepsilon}^{s, \tau}(x)=G_{\varepsilon}^{s, t}(x)+G_{t-s+\varepsilon}^{t, \tau}(x) .
$$

Следующий пример показывает, что обычные аддитивные функционалы можно записать в терминах определения 2.4.

П р и м е р 2.2. Пусть $f: \mathbf{R} \rightarrow \mathbf{R}$ - ограниченная борелевская функция. Тогда функционал

$$
\varphi_{t}^{s}(x)=\int_{s}^{t} f(x+w(r)-w(s)) d r, \quad x \in \mathbf{R}, \quad 0 \leqslant s<t \leqslant 1,
$$

является однородным аддитивным функционалом от $\widetilde{w}$ [1].

Определим обобщенный аддитивный функционал $G$ нулевого порядка по формуле для любых $0<\varepsilon<s<t \leqslant 1, x \in \mathbf{R}$

$$
G_{\varepsilon}^{s, t}(x)=\int_{s}^{t} f(x+w(r)-w(s-\varepsilon)) d r .
$$

Проверим, что $G$ удовлетворяет условиям определения 2.4. Условие 1) выполнено. Сейчас $G_{\varepsilon}^{s, t}(x)$ - интегрируемая с квадратом случайная величина, измеримая относительно $\mathscr{F}^{s-\varepsilon, t}$. Условие 2$)$ сейчас $(p=0)$ превращается в верное соотношение. Действительно, если $G_{\delta}^{s, t}(y)=$ $\sum_{k=0}^{\infty} I_{k}^{s-\delta, t}\left(a_{k}(y, \delta, s, t)\right)$, то

$$
\sum_{k=0}^{\infty} k !\left\|a_{k}(y, \delta, s, t)\right\|_{k}^{2}=\mathbf{E}\left(\int_{s}^{t} f(y+w(r)-w(s-\delta)) d r\right)^{2} \leqslant C^{2}
$$


где $C=\sup _{\mathbf{R}}|f|$. Поэтому

$$
\mathbf{E} \sum_{k=0}^{\infty} k !\left\|a_{k}(x+w(s-\delta)-w(s-\varepsilon), \delta, s, t)\right\|_{k}^{2} \leqslant C^{2} .
$$

Условия 3) и 5) выполнены очевидным образом. Условие 4)выполняется в силу рассуждений примера 2.1. Таким образом, обычный аддитивный однородный функционал $\varphi$ можно отождествить сейчас с обобщенным функционалом $G$.

В приведенном примере отступление на $\varepsilon$ кажется искусственным, однако, как будет показано в следующем пункте, оно необходимо, если $f$ заменить обобщенной функцией ненулевого порядка.

3. Некоторые интегралы, связанные с винеровским процессом. В этом пункте интегралы типа $\int_{0}^{t} \varkappa(w(s)) d s$, где $\varkappa \in S^{*}-$ обобщенная функция медленного роста, определяются как обобщенные случайные величины из $W_{-p}^{0, t}$ и других пространств.

Определим вначале $\varkappa(w(t)), t \in(0 ; 1]$. Так как $\varkappa(\cdot / \sqrt{t})$ также является элементом $S^{*}$ того же порядка, что $\varkappa$, то достаточно определить $\varkappa(w(1))$, а затем произвести очевидный пересчет.

О п р е д е л е н и е 3.1 . Для обобщенной функции $\varkappa \in S^{*}$ порядка $m \geqslant 0 \varkappa(w(1))$ - это элемент $W_{-m-1}^{0,1}$, имеющий разложение Ито-Винера

$$
\varkappa(w(1))=\sum_{k=0}^{\infty} \frac{1}{k !} I_{k}^{0,1}\left(\mathbb{1}_{[0 ; 1]}^{\otimes k}\right)\left\langle\varkappa, H_{k}(\cdot) p_{1}(\cdot)\right\rangle .
$$

Лемма 3.1. Ряд (3.1) сходится в $W_{-m-1}^{0,1}$.

Д о к а з а т е л ь с т в о. Имеем

$$
\begin{aligned}
& \sum_{k=0}^{\infty}(k+1)^{-m-1}(k !)^{-1}\left\langle\varkappa, H_{k}(\cdot) p_{1}(\cdot)\right\rangle^{2} \\
& \leqslant \sum_{k=0}^{\infty}(k !)^{-1}(k+1)^{-m-1}\|\varkappa\|_{-m}^{2} \\
& \quad \times\left[\sup _{x \in \mathbf{R}, \alpha \leqslant m}\left|\frac{d^{\alpha}}{d x^{\alpha}}\left(H_{k}(x) e^{-x^{2} / 2} \frac{1}{\sqrt{2 \pi}}\right)\left(1+x^{2}\right)^{m / 2}\right|\right]^{2} \\
& =\sum_{k=0}^{\infty}(k+1)^{-m-1}\|\varkappa\|_{-m}^{2} \\
& \quad \times\left[\sup _{x \in \mathbf{R}, \alpha \leqslant m}\left|\frac{\sqrt{(k+\alpha) !}}{\sqrt{k !}} \frac{H_{k+\alpha}(x)}{\sqrt{(k+\alpha) !}} e^{-x^{2} / 2} \frac{1}{\sqrt{2 \pi}}\left(1+x^{2}\right)^{m / 2}\right|\right]^{2} \\
& \leqslant \sum_{k=0}^{\infty}(k+1)^{-m-1}\|\varkappa\|_{-m}^{2}(k+m)^{m} C_{1}\left[\sup _{x \in \mathbf{R}, n \geqslant k}\left|\frac{H_{n}(x)}{\sqrt{n !}} e^{-x^{2} / 4}\right|\right]^{2} \\
& \leqslant \sum_{k=0}^{\infty}\|\varkappa\|_{-m}^{2} C_{1} C_{2}^{2}(k+1)^{-m-1}(k+m)^{m} k^{-1 / 6}<+\infty .
\end{aligned}
$$


Здесь $C_{1}=\left[\sup _{x \in \mathbf{R}}(\sqrt{2 \pi})^{-1}\left(1+x^{2}\right)^{m / 2} e^{-x^{2} / 4}\right]^{2}$, а константа $C_{2}$ взята из оценки (см. [10]):

$$
\sup _{x \in \mathbf{R}} \frac{1}{\sqrt{n !}}\left|H_{n}(x) e^{-x^{2} / 4}\right| \leqslant C_{2} n^{-1 / 12}, \quad n \geqslant 1 .
$$

Лемма доказана.

Отметим, что обобщенная случайная величина $\varkappa(w(1))$ может быть в некоторых случаях получена как предел в $W_{-m-1}^{0,1}$ обычных случайных величин $\varkappa_{\varepsilon}(w(1))$ при $\varepsilon \rightarrow+0$, где $\varkappa_{\varepsilon}-$ «сглаживание» $\varkappa$, т.е. свертка c $p_{\varepsilon}$.

Докажем данное утверждение для функций $\varkappa \in S^{*}$, являющихся обобщенными производными функций из $L_{2}(\mathbf{R})$.

Лемма 3.2. Пусть $\varkappa=D^{m} f, f \in L_{2}(\mathbf{R}) \cap L_{1}(\mathbf{R}), m \geqslant 0$, m.e.

$$
\langle\varkappa, \varphi\rangle=(-1)^{m} \int_{\mathbf{R}} f(x) \frac{d^{m}}{d x^{m}} \varphi(x) d x, \quad \varphi \in S .
$$

Тогда $\varkappa_{\varepsilon}(w(1)) \rightarrow \varkappa(w(1)), \varepsilon \rightarrow+0$, в $W_{-m-1}^{0,1}$.

Д ок а з а т е ль с т в о. Аналогично разложению $\varkappa(w(1))$ ряд Ито-Винера для $\varkappa_{\varepsilon}(w(1))$ имеет вид

$$
\varkappa_{\varepsilon}(w(1))=\sum_{k=0}^{\infty} \frac{1}{k !} I_{k}^{0,1}\left(\mathbb{I}_{[0 ; 1]}^{\otimes k}\right)(1+\varepsilon)^{-k / 2}\left\langle\varkappa, H_{k}\left(\frac{\cdot}{\sqrt{1+\varepsilon}}\right) p_{1+\varepsilon}(\cdot)\right\rangle .
$$

Подставляя $\varkappa=D^{m} f$, получим

$$
\varkappa_{\varepsilon}(w(1))=\sum_{k=0}^{\infty} \frac{1}{k !} I_{k}^{0,1}\left(\mathbb{I}_{[0 ; 1]}^{\otimes k}\right)(1+\varepsilon)^{-(k+m) / 2}\left\langle f, H_{k+m}\left(\frac{\cdot}{\sqrt{1+\varepsilon}}\right) p_{1+\varepsilon}(\cdot)\right\rangle .
$$

Следовательно,

$$
\begin{aligned}
& \left\|\varkappa(w(1))-\varkappa_{\varepsilon}(w(1))\right\|_{-m-1}^{2} \\
& \begin{array}{l}
=\sum_{k=0}^{\infty}(k+1)^{-m-1}\left(\left\langlef, H_{k+m}(\cdot) p_{1}(\cdot)-(1+\varepsilon)^{-(k+m) / 2}\right.\right. \\
\left.\left.\quad \times H_{k+m}\left(\frac{\cdot}{\sqrt{1+\varepsilon}}\right) p_{1+\varepsilon}(\cdot)\right\rangle\right)^{2} \frac{1}{k !} \\
\leqslant 2 \sum_{k=0}^{\infty} \frac{(k+m)^{m}}{(k+1)^{m+1}}\left(\left\langle f, H_{k+m}(\cdot) p_{1}(\cdot)\right\rangle\right)^{2}\left(1-(1+\varepsilon)^{-(k+m) / 2}\right)^{2} \frac{1}{(k+m) !} \\
+2 \sum_{k=0}^{\infty} \frac{(k+m)^{m}}{(k+1)^{m+1}}(1+\varepsilon)^{-(k+m)} \\
\quad \times\left(\left\langle f, H_{k+m}(\cdot) p_{1}(\cdot)-H_{k+m}\left(\frac{\cdot}{\sqrt{1+\varepsilon}}\right) p_{1+\varepsilon}(\cdot)\right\rangle\right)^{2} \frac{1}{(k+m) !} .
\end{array}
\end{aligned}
$$


Так как $((k+m) !)^{-1}\left(\left\langle f, H_{k+m}(\cdot) p_{1}(\cdot)\right\rangle\right)^{2} \leqslant C_{3}^{2}(2 \pi)^{-1}\|f\|^{2}(k+m)^{-1 / 6}$, где использовано неравенство (см. [10]) $\sup _{x \in \mathbf{R}}\left|H_{n}(x)(n !)^{-1 / 2} e^{-x^{2} / 2}\right| \leqslant$ $C_{3} n^{-1 / 12}, n>0$, то первый ряд последней суммы, очевидно, стремится к нулю при $\varepsilon \rightarrow+0$.

Перейдем к оценке второго ряда. Обозначим

$$
\begin{aligned}
a_{k+m, \varepsilon}^{2} & :=\left(\left\langle f, H_{k+m}(\cdot) p_{1}(\cdot)-H_{k+m}\left(\frac{\cdot}{\sqrt{1+\varepsilon}}\right) p_{1+\varepsilon}(\cdot)\right\rangle\right)^{2} \frac{1}{(k+m) !} \\
& =\left(\int_{\mathbf{R}}(f(x)-f(\sqrt{1+\varepsilon} x)) H_{k+m}(x) p_{1}(x) d x\right)^{2} \frac{1}{(k+m) !} .
\end{aligned}
$$

Очевидно, что ряд

$$
\sum_{k=0}^{\infty} a_{k, \varepsilon}^{2}=\int_{\mathbf{R}}(f(x)-f(\sqrt{1+\varepsilon} x))^{2} p_{1}(x) d x=\|f(x)-f(\sqrt{1+\varepsilon} x)\|^{2}
$$

составляет квадрат нормы функции $f(x)-f(\sqrt{1+\varepsilon} x), x \in \mathbf{R}$, в пространстве $L_{2}\left(\mathbf{R}, e^{-x^{2} / 2} / \sqrt{2 \pi} d x\right)$. Обычным образом можно проверить, что $\|f(x)-f(\sqrt{1+\varepsilon} x)\|^{2} \rightarrow 0$ при $\varepsilon \rightarrow+0$. Следовательно, и второй ряд в оценке квадрата нормы разности $\varkappa(w(1))-\varkappa_{\varepsilon}(w(1))$ стремится к нулю при $\varepsilon \rightarrow+0$, что и требовалось доказать.

Обозначим через $A$ самосопряженное замыкание в $L_{2}(\mathbf{R})$ оператора

$$
(A f)(x)=-f^{\prime \prime}(x)+\left(1+x^{2}\right) f(x), \quad x \in \mathbf{R}, \quad f \in S .
$$

С помощью «сглаживающего» оператора $A$ построим шкалу пространств вида

$$
\widetilde{S}_{p}:=\left\{\varphi \sim\left(\varphi_{k}\right):\|\varphi\|_{2, p}^{2}=\sum_{k=0}^{\infty} k !\left\|\left(A^{p}\right)^{\otimes n} \varphi_{n}\right\|_{n}^{2}<+\infty\right\}, \quad p \in \mathbf{R},
$$

где $\varphi \sim\left(\varphi_{k}\right)$ - краткое обозначение разложения случайной величины $\varphi$ по кратным интегралам Ито-Винера с симметричными ядрами $\varphi_{n} \in$ $L_{2}\left([0 ; 1]^{n}\right) \subset L_{2}\left(\mathbf{R}^{n}\right)$.

Пространство $\widetilde{S}=\bigcap_{p \geqslant 0} \widetilde{S}_{p}$ является ядерным (с соответствующей системой норм $\left.\left\{\|\cdot\|_{2, p}, p \geqslant 0\right\}\right)$ и его сопряженное пространство есть

$$
\widetilde{S}^{*}=\bigcup_{p \geqslant 0} \widetilde{S}_{-p}
$$

Элементы пространства $\widetilde{S}^{*}$ называют распределениями Хида.

Перейдем теперь к рассмотрению функционалов вида

$$
L(t, \varkappa)=\int_{0}^{t} \varkappa(w(s)) d s .
$$

Так как ядра разложения Ито-Винера для функции $\varkappa(w(t))$ имеют особенность по переменной $t$ в точке $t=0$, то в общем случае трудно ожидать, чтобы функционал $L(t, \varkappa)$ был обобценным винеровским функционалом, и, следовательно, требуется некоторая его перенормировка. 
Теорема 3.1. Пусть $\varkappa \in S^{*}-$ обобщенная функиия порядка $m \geqslant 0 u \varkappa(w(s)) \sim\left(\varphi_{k}(s)\right), 0<s \leqslant 1, t \in(0 ; 1]$. Tогда cyществует такое $q_{0}>0$, что для всех $p>q_{0}$ функционал вида

$$
\widehat{L}(t, \varkappa):=\int_{0}^{t}\left(\varkappa(w(s))-\sum_{k=0}^{m-1} I_{k}^{0, s}\left(\varphi_{k}(s)\right)\right) d s
$$

является элементом $\widetilde{S}_{-p}$.

Док аз а те ль с тв о. Так как

$$
\left\|A^{-p} \mathbb{1}_{[0 ; s]}\right\|_{2}^{2}=\sum_{k=0}^{\infty}(2 k+2)^{-p}\left|\left\langle\mathbb{I}_{[0 ; s]}, e_{n}\right\rangle\right|^{2} \leqslant s^{2} C_{4}^{2} \sum_{k=0}^{\infty}(2 k+2)^{-p}
$$

где $C_{4}=\sup _{x \in \mathbf{R}, n \geqslant 0}\left|e_{n}(x)\right|$, то найдется такое число $q_{0}>0$, что $\left\|A^{-q_{0}} \mathbb{I}_{[0 ; s]}\right\|_{2}^{2} \leqslant s^{2}$. Здесь $\left\{e_{n} ; n \geqslant 1\right\}-$ функции Эрмита $e_{n}(x)=$ $(-1)^{n}(n !)^{-1 / 2}(2 \sqrt{\pi})^{-n / 2} e^{x^{2} / 2}(d / d x)^{n} e^{-x^{2}}$, образующие собственный ортонормированный базис оператора $A$ в $L_{2}(\mathbf{R})$.

Поэтому, учитывая, что симметричные ядра разложения функционала $\varkappa(w(s))$ в кратные интегралы Ито-Винера имеют вид

$$
\varphi_{k}(s)=\frac{1}{k !} \mathbb{I}_{[0 ; s]}^{\otimes k} s^{-k / 2}\left\langle\varkappa, H_{k}\left(\frac{\cdot}{\sqrt{s}}\right) p_{s}(\cdot)\right\rangle,
$$

получим следующую оценку:

$$
\begin{aligned}
& \left\|\varkappa(w(s))-\sum_{k=0}^{m-1} I_{k}^{0, s}\left(\varphi_{k}(s)\right)\right\|_{2,-p}^{2} \\
& =\sum_{k=m}^{\infty} k !\left\|(A)^{\otimes k}\left(\mathbb{I}_{[0 ; s]}^{\otimes k}\right)\right\|_{2}^{2} \frac{s^{k}}{(k !)^{2}}\left|\left\langle\varkappa, H_{k}\left(\frac{\cdot}{\sqrt{s}}\right) p_{s}(\cdot)\right\rangle\right|^{2} \\
& \leqslant \sum_{k=m}^{\infty} 2^{-2 k\left(p-q_{0}\right)} s^{k} \frac{1}{k !}\left|\left\langle\varkappa, H_{k}\left(\frac{\cdot}{\sqrt{s}}\right) p_{s}(\cdot)\right\rangle\right|^{2} .
\end{aligned}
$$

Действуя так же, как и при доказательстве леммы 3.1 , легко получить неравенство

$$
\begin{aligned}
& \frac{1}{\sqrt{k !}}\left|\left\langle\varkappa, H_{k}\left(\frac{\cdot}{\sqrt{s}}\right) p_{s}(\cdot)\right\rangle\right| \\
& \quad \leqslant C_{2}^{2}\|\varkappa\|_{-m}^{2} \frac{(k+m)^{m}}{2 \pi s^{m+1}} k^{-1 / 6} \sup _{s \in[0 ; 1]}\left(1+x^{2}\right)^{m} e^{-x^{2} /(2 s)} .
\end{aligned}
$$

Исследуя функцию $g(x)=\left(1+x^{2}\right)^{m} e^{-x^{2} /(2 s)}, x \in \mathbf{R}$, легко показать, что она равномерно ограничена по $s \in(0 ; 1]$, т.е.

$$
\sup _{x \in \mathbf{R}, s \in(0 ; 1]} g(x) \leqslant C_{5}=C_{5}(m) .
$$


Следовательно,

$$
\begin{aligned}
& \left\|\varkappa(w(s))-\sum_{k=0}^{m} I_{k}^{0, s}\left(\varphi_{k}(s)\right)\right\|_{2,-\left(p+q_{0}\right)}^{2} \\
& \leqslant C_{5} C_{2}^{2} \frac{\|\varkappa\|_{-m}^{2}}{2 \pi} \sum_{k=m}^{\infty} 2^{-2 n\left(p-q_{0}\right)} s^{k-m-1}(k+m)^{m} k^{-1 / 6} .
\end{aligned}
$$

Таким образом, для произвольного $p>q_{0}$

$$
\int_{0}^{t}\left\|\varkappa(w(s))-\sum_{k=0}^{m} I_{k}^{0, s}\left(\varphi_{k}(s)\right)\right\|_{2,-p} d s<+\infty
$$

откуда следует утверждение теоремы.

Выбирая обобщенные функции $\varkappa \in S^{*}$ так, чтобы происходило сглаживание ядер $\varphi_{n}(s)$ в точке $s=0$, можно получить функционалы $L(t, \varkappa)$, которые не требуют перенормировки.

Теорема 3.2. Пусть функиия $\varkappa \in S^{*}$ есть обобщенная производная порядка $m \geqslant 0$ некоторой функиии $f \in L_{2}(\mathbf{R}) \cap L_{1}(\mathbf{R})$ такой, что сушествуют производные $f(0)=f^{\prime}(0)=\cdots=f^{(m-1)}(0)=0$. Тогда для произвольных $t \in[0 ; 1], p>0$

$$
L(t, \varkappa) \in \widetilde{S}_{-p}
$$

Д о к а за т е л с с т о. Легко видеть, что

$$
\begin{aligned}
\|\varkappa(w(s))\|_{2,-p}^{2} & \leqslant \sum_{k=0}^{\infty} 2^{-2 k p} \frac{1}{k !}\left|\left\langle D^{m} f, H_{k}\left(\frac{\cdot}{\sqrt{s}}\right) p_{s}(\cdot)\right\rangle\right|^{2} \\
& \leqslant \sum_{k=0}^{\infty} 2^{-2 n k} \frac{(k+m)^{m}}{s^{m}}\left|\left\langle f, H_{k+m}\left(\frac{\cdot}{\sqrt{s}}\right) p_{s}(\cdot)\right\rangle\right|^{2} \frac{1}{(k+m) !} .
\end{aligned}
$$

Обозначим

$$
a_{k}(s):=\left\langle f, H_{k}\left(\frac{\cdot}{\sqrt{s}}\right) p_{s}(\cdot)\right\rangle \frac{1}{\sqrt{k !}}=\int_{\mathbf{R}} f(\sqrt{s} x) H_{k}(x) p_{1}(x) d x \frac{1}{\sqrt{k !}} .
$$

Так как

$$
\frac{1}{s^{m}} \sum_{k=0}^{\infty} a_{k}^{2}(s)=\frac{1}{s^{m}} \int_{\mathbf{R}} f^{2}(\sqrt{s} x) \frac{e^{-x^{2} / 2}}{\sqrt{2 \pi}} d x \leqslant \frac{C_{6}}{s}
$$

при достаточно малых $s>0$, то, следовательно,

$$
\int_{0}^{t}\|\varkappa(w(s))\|_{2,-p} d s<+\infty, \quad p>0, \quad t \in[0 ; 1],
$$

и, таким образом, $L(t, \varkappa)=\int_{0}^{t} \varkappa(w(s)) d s \in \widetilde{S}_{-p}, p>0$. Теорема доказана.

Приведенные утверждения показывают, что «локальные времена», соответствующие обобщенным функциям из $S^{*}$, не могут быть обобщенными винеровскими функционалами из-за особенности ядер разложения 
Ито-Винера в точке 0. Именно это обстоятельство мотивировало отступление на $\varepsilon>0$ в основном определении 2.4. Как показывает следующий пункт, при этом удается сохранить все хорошие свойства обычных аддитивных функционалов.

4. Восстановление обобщенного аддитивного функционала по его характеристике. Пусть $G=\left\{G_{\varepsilon}^{s, t} ; 0<\varepsilon<s<t \leqslant 1\right\}$, как и в предыдущих пунктах, обобщенный аддитивный однородный функционал от винеровского процесса порядка $m$. Пусть

$$
G_{\varepsilon}^{s, t}(x)=\sum_{k=0}^{\infty} I_{k}^{s-\varepsilon, t}\left(a_{k}(x, \varepsilon, s, t)\right)
$$

- разложение Ито-Винера для $G$. Аналогом характеристики функционала $G$ сейчас является $a_{0}(x, \varepsilon, s, t), x \in \mathbf{R}$ (это слагаемое соответствует математическому ожиданию в разложении Ито-Винера квадратичноинтегрируемой случайной величины). Цель настоящего пункта - восстановить $G$ по его характеристике и определить обобщенную функцию $\varkappa$, с помощью которой $G$ задается как обобщенное локальное время, т.е. корректно определенный аналог интеграла (1.1).

Теорема 4.1. Пусть $G_{\varepsilon}^{s, t}(x)$ при фиксированном $x$ имеет в пространстве $W_{-m}^{0,1}$ непрерывную на $0<\varepsilon<s<t \leqslant 1$ производную по $t$ $g_{\varepsilon}^{s, t}(x)$. Пусть нулевой член разложения Ито-Винера для $g_{\varepsilon}^{s, t}(x)$

$$
\alpha_{0}(x, \varepsilon, s, t)=\frac{d}{d t} a_{0}(x, \varepsilon, s, t)
$$

удовлетворяет условию

$$
\exists C>0: \forall \varphi \in S: \quad\left|\int_{\mathbf{R}} \alpha_{0}(x, \varepsilon, s, t) \varphi(x) d x\right| \leqslant C\|\varphi\|_{-m} .
$$

Тогда функционал $G$ является обобщенным локальным временем, отвечаюшим некоторой функиии $\varkappa \in S_{-m}$.

Д о к а з а т е л ь с т в о. Так как $G$-однородный функционал, то для любых $0<\varepsilon<s<t \leqslant 1, x \in \mathbf{R}$ и $h \in(0, s-\varepsilon)$

$$
a_{0}(x, \varepsilon, s, t)=a_{0}(x, \varepsilon, s-h, t-h) .
$$

Кроме того, для любых $0<\varepsilon_{1}<\varepsilon<s<t \leqslant 1, x \in \mathbf{R}$

$$
a_{0}(x, \varepsilon, s, t)=a_{0}\left(\cdot, \varepsilon_{1}, s, t\right) * p_{\varepsilon-\varepsilon_{1}}(x),
$$

где, как и раньше, $p_{\varepsilon-\varepsilon_{1}}$ - переходная плотность винеровского процесса за время $\varepsilon-\varepsilon_{1}$. Поэтому, в силу аддитивности $G$, производная $\alpha_{0}(x, \varepsilon, s, t)$ зависит лишь от $t-s+\varepsilon$. Обозначим $\alpha_{0}(x, \varepsilon, s, t)$ через $f(x, t-s+\varepsilon)$. Функция $f$ на $\mathbf{R} \times(0 ; 1]$ удовлетворяет уравнению 
теплопроводности

$$
\frac{\partial f}{\partial t}=\frac{1}{2} \frac{\partial^{2} f}{\partial x^{2}}
$$

Рассмотрим последовательность $\left\{t_{n} ; n \geqslant 1\right\}$ точек из $(0 ; 1]$ такую, что $\lim _{n \rightarrow \infty} t_{n}=0$. Функции $\left\{f\left(\cdot, t_{n}\right) ; n \geqslant 1\right\}$ как элементы $S_{-m}$ образуют ограниченную последовательность. По теореме Банаха-Алаоглу существует подпоследовательность $\left\{f\left(\cdot, t_{n(k)}\right) ; k \geqslant 1\right\}$, слабо сходяшаяся к некоторому $\varkappa \in S_{-m}$. Докажем, что

$$
\forall t \in(0 ; 1]: f(x, t)=\varkappa * p_{t}(x), \quad x \in \mathbf{R} .
$$

Действительно, при достаточно больших номерах $k$

$$
\begin{aligned}
\forall \varphi \in S_{m}: \int_{\mathbf{R}} \varphi(x) f(x, t) d x & =\int_{\mathbf{R}} \varphi(x) p_{t-t_{n(k)}} * f\left(\cdot, t_{n(k)}\right)(x) d x \\
& =\int_{\mathbf{R}} \varphi * p_{t-t_{n(k)}}(y) f\left(y, t_{n(k)}\right) d y .
\end{aligned}
$$

При этом свертка $\varphi * p_{t-t_{n(k)}}$ сходится к $\varphi * p_{t}$ при $k \rightarrow \infty$ в $S_{m}$. Следовательно,

$$
\int_{\mathbf{R}} \varphi * p_{t-t_{n(k)}}(y) f\left(y, t_{n(k)}\right) d y \longrightarrow\left\langle\varphi * p_{t}, \varkappa\right\rangle, \quad k \rightarrow \infty .
$$

Согласно свойствам свертки в $S_{-m}$, имеем $\left\langle\varphi * p_{t}, \varkappa\right\rangle=\left\langle\varphi, \varkappa * p_{t}\right\rangle$. Окончательно,

$$
\int_{\mathbf{R}} \varphi(x) f(x, t) d x=\left\langle\varphi, \varkappa * p_{t}\right\rangle, \quad \varphi \in S_{m} .
$$

Поэтому $f(\cdot, t)=\varkappa * p_{t}$ как элемент пространства $S_{-m}$. Проверим теперь, что функционал $G$ задается с помощью функции $\varkappa$ как обобщенное локальное время. Для этого достаточно проверить следуюшие два соотношения: для любых $0<\varepsilon<s<t \leqslant 1, x \in \mathbf{R}$

$$
\begin{gathered}
G_{\varepsilon}^{s, t}=\int_{s}^{t} g_{\varepsilon}^{s, r} d r \\
g_{\varepsilon}^{s, r}(x)=\varkappa(x+w(r)-w(s-\varepsilon)) .
\end{gathered}
$$

Соотношение (4.1) получается стандартным образом с учетом непрерывности производной $\frac{d}{d t} G_{\varepsilon}^{s, t}(x)=g_{\varepsilon}^{s, t}(x)$ в гильбертовом пространстве $W_{-m}^{0,1}$. Проверим равенство (4.2). Согласно свойствам $G$,

$$
\begin{aligned}
g_{\varepsilon}^{s, t}(x) & =\lim _{\Delta \rightarrow+0} \frac{1}{\Delta}\left[G_{\varepsilon}^{s, t+\Delta}(x)-G_{\varepsilon}^{s, t}(x)\right] \\
& =\lim _{\Delta \rightarrow+0} \frac{1}{\Delta}\left[G_{\varepsilon}^{s, t}(x)+G_{t-s+\varepsilon}^{t, t+\Delta}(x)-G_{\varepsilon}^{s, t}(x)\right]=\lim _{\Delta \rightarrow+0} \frac{1}{\Delta} G_{t-s+\varepsilon}^{t, t+\Delta}(x) .
\end{aligned}
$$

Поэтому для всякого $\delta \in(0 ; t-s+\varepsilon)$

$$
g_{\varepsilon}^{s, t}(x)=\lim _{\Delta \rightarrow+0} \frac{1}{\Delta} G_{\delta}^{t, t+\Delta}(x+w(t-\delta)-w(s-\varepsilon)) .
$$


Согласно определению действия случайного отображения, из (4.3) следует, что ядра слагаемых из разложения Ито-Винера для $g_{\varepsilon}^{s, t}(x)$ являются постоянными на соответствуюших степенях отрезка $[s-\varepsilon ; t]$. Теперь, с учетом равенства

$$
g_{\varepsilon}^{s, t}(x)=g_{\delta}^{t-\delta, t}(x+w(t-2 \delta)-w(s-\varepsilon)),
$$

получаем, что

$$
g_{\varepsilon}^{s, t}(x)=f(x+w(t-2 \delta)-w(s-\varepsilon), 2 \delta), \quad x \in \mathbf{R} .
$$

Переходя к пределу при $\delta \rightarrow+0$, получим

$$
g_{\varepsilon}^{s, t}(x)=\varkappa(x+w(t)-w(s-\varepsilon)), \quad x \in \mathbf{R} .
$$

Теорема доказана.

П р и м е р 4.1. Локальное время в нуле как обобщенный аддитивный функционал. Рассмотрим аддитивный функционал от $w$, который задается соотношением

$$
L(x, s, t)=\lim _{\varepsilon \rightarrow+0} \frac{1}{2 \varepsilon} \int_{s}^{t} \mathbb{I}_{[-\varepsilon ; \varepsilon]}(x+w(r)-w(s)) d r
$$

где предел понимается в смысле сходимости по вероятности. Известно [11], что $L(x, s, t)$ можно переписать в виде

$$
\begin{aligned}
L(x, s, t)= & 2(x+w(t)-w(s))_{+}-2(x)_{+} \\
& -2 \int_{s}^{t} \mathbb{I}_{[0 ;+\infty)}(x+w(r)-w(s)) d w(r) .
\end{aligned}
$$

Из этой формулы следует, что отображение $G_{\varepsilon}^{s, t}(x)=L(x+w(s)-w(s-$ $\varepsilon), s, t)$ является обобщенным аддитивным функционалом от $w$ нулевого порядка, т.е. принимающим значения в $L_{2}(\Omega, \mathscr{F}, \mathbf{P})$. Проверим, что $G$ удовлетворяет условиям теоремы 4.1 для $m=1$. Для этого запишем разложение Ито-Винера для $G_{\varepsilon}^{s, t}(x)$. Найдем сначала разложение ИтоВинера отдельных слагаемых из (4.4).

Для любого $x \in \mathbf{R}$ и любого $t \in(0 ; 1]$

$$
\begin{aligned}
(x+w(t))_{+} & =\sum_{k=0}^{\infty} \frac{1}{k !} \int_{\mathbf{R}}(x+u)_{+} H_{k}\left(\frac{u}{\sqrt{t}}\right) p_{t}(u) d u H_{k}\left(\frac{w(t)}{\sqrt{t}}\right) \\
= & \sum_{k=0}^{\infty} \frac{1}{k !} \frac{1}{\sqrt{t}} \int_{\mathbf{R}}(x+u)_{+} H_{k}\left(\frac{u}{\sqrt{t}}\right) p_{t}(u) d u I_{k}^{0, t}\left(\mathbb{I}_{[0 ; t]}^{\otimes k}\right), \\
\mathbb{I}_{[0 ;+\infty)}(x+w(t))= & \sum_{k=0}^{\infty} \frac{1}{k !} \frac{1}{(\sqrt{t})^{k}} \\
& \times \int_{\mathbf{R}} \mathbb{I}_{[0 ;+\infty)}(x+u) H_{k}\left(\frac{u}{\sqrt{t}}\right) p_{t}(u) d u I_{k}^{0, t}\left(\mathbb{I}_{[0 ; t]}^{\otimes k}\right),
\end{aligned}
$$




$$
\begin{aligned}
& \int_{s}^{t} \mathbb{I}_{[0 ;+\infty)}(x+w(r)-w(s-\varepsilon)) d w(r) \\
& =\sum_{k=0}^{\infty} \frac{1}{k !} \int_{s}^{t} \frac{1}{(\sqrt{r-s+\varepsilon})^{k}} \int_{\mathbf{R}} \mathbb{I}_{[0 ;+\infty)}(x+u) H_{k}\left(\frac{u}{\sqrt{r-s+\varepsilon}}\right) \\
& \quad \times p_{r-s+\varepsilon}(u) d u I_{k}^{s-\varepsilon, r}\left(\mathbb{I}_{[s-\varepsilon ; r]}^{\otimes k}\right) d w(r) \\
& =\sum_{k=0}^{\infty} \frac{1}{(k+1) !} I_{k+1}^{s-\varepsilon, t}\left(\mathbb{I}_{\left\{r^{*} \in[s ; t]\right\}} \mathbb{I}_{\left\{r_{*} \in[s-\varepsilon ; t]\right\}} \frac{1}{\left(\sqrt{r^{*}-s+\varepsilon}\right)^{k}}\right. \\
& \left.\quad \times \int_{\mathbf{R}} \mathbb{I}_{[0 ;+\infty)}(x+u) H_{k}\left(\frac{u}{\sqrt{r^{*}-s+\varepsilon}}\right) p_{r^{*}-s+\varepsilon}(u) d u\right),
\end{aligned}
$$

где $r_{*}=\min _{i=1, \ldots, k+1} r_{i}, r^{*}=\max _{i=1, \ldots, k+1} r_{i}$.

Поэтому

$$
\begin{aligned}
G_{\varepsilon}^{s, t}(x)=2 \sum_{k=0}^{\infty} \frac{1}{k !}[ & \frac{1}{(\sqrt{t-s+\varepsilon})^{k}} I_{k}^{s-\varepsilon, t}\left(\mathbb{I}_{[s-\varepsilon ; t]}^{\otimes k}\right) \\
& \times \int_{\mathbf{R}}(x+u)_{+} H_{k}\left(\frac{u}{\sqrt{t-s+\varepsilon}}\right) p_{t-s+\varepsilon}(u) d u \\
& \left.\quad-\frac{1}{\sqrt{\varepsilon}^{k}} I_{k}^{s-\varepsilon, s}\left(\mathbb{I}_{[s-\varepsilon ; s]}^{\otimes k}\right) \int_{\mathbf{R}}(x+u)_{+} H_{k}\left(\frac{u}{\sqrt{\varepsilon}}\right) p_{\varepsilon}(u) d u\right] \\
-2 \sum_{k=1}^{\infty} \frac{1}{k !} I_{k}^{s-\varepsilon, t}\left(\mathbb{I}_{\left\{r^{*} \in[s ; t]\right\}} \mathbb{I}_{\left\{r_{*} \in[s-\varepsilon ; t]\right\}} \frac{1}{\left(\sqrt{r^{*}-s+\varepsilon}\right)^{k-1}}\right. & \left.\times \int_{\mathbf{R}} \mathbb{I}_{[0 ;+\infty)}(x+u) H_{k-1}\left(\frac{u}{\sqrt{r^{*}-s+\varepsilon}}\right) p_{r^{*}-s+\varepsilon}(u) d u\right) .
\end{aligned}
$$

При фиксированных $k \geqslant 1 k$-е ядро из разложения Ито-Винера для функционала $G_{\varepsilon}^{s, t}(x)$ имеет вид

$$
\begin{aligned}
& 2 \mathbb{I}_{\left\{r^{*} \in[s ; t]\right\}} \mathbb{I}_{\left\{r_{*} \in[s-\varepsilon ; t]\right\}} \\
& \times\left(\frac{1}{k !} \frac{1}{(\sqrt{t-s+\varepsilon})^{k}} \int_{\mathbf{R}}(x+u)_{+} H_{k}\left(\frac{u}{\sqrt{t-s+\varepsilon}}\right) p_{t-s+\varepsilon}(u) d u\right. \\
& \quad-\frac{1}{(k-1) !} \frac{1}{\left(\sqrt{r^{*}-s+\varepsilon}\right)^{k-1}} \\
& \left.\quad \times \int_{\mathbf{R}} \mathbb{I}_{[0 ;+\infty)}(x+u) H_{k-1}\left(\frac{u}{\sqrt{r^{*}-s+\varepsilon}}\right) p_{r^{*}-s+\varepsilon}(u) d u\right) \\
& -\frac{2}{k !} \frac{1}{(\sqrt{\varepsilon})^{k}} \mathbb{I}_{[s-\varepsilon ; s]}^{\otimes k} \int_{\mathbf{R}}(x+u)_{+} H_{k}\left(\frac{u}{\sqrt{\varepsilon}}\right) p_{\varepsilon}(u) d u .
\end{aligned}
$$

Для дальнейших рассуждений нам понадобится следующая лемма. 
Лемма 4.1. Пусть $f \in C^{1}([0 ; 1]), k \geqslant 1$. Тогда $L_{2}\left([0 ; 1]^{k}\right)$-значная бункиия $h_{k}(t), t \in[0 ; 1]$, заданная по правилу

$$
\begin{aligned}
& h_{k}(t)\left(\tau_{1}, \ldots, \tau_{k}\right)=\mathbb{I}_{[0 ; t]}^{\otimes k}\left(\tau_{1}, \ldots, \tau_{k}\right)\left[f(t)-f\left(\tau^{*}\right)\right], \\
& \tau_{1}, \ldots, \tau_{k} \in[0 ; 1], \quad \tau^{*}=\max \left\{\tau_{i} ; i=1, \ldots, n\right\}
\end{aligned}
$$

непрерывно дифференцируема по $t$, причем

$$
h_{k}^{\prime}(t)\left(\tau_{1}, \ldots, \tau_{k}\right)=\mathbb{I}_{[0 ; t]}^{\otimes k}\left(\tau_{1}, \ldots, \tau_{k}\right) f^{\prime}(t) .
$$

Д ок а з а т е л ь с т в о. Рассмотрим $\lim _{\Delta \rightarrow-0} \Delta^{-1}(h(t+\Delta)-h(t))$ для $t \in(0 ; 1]$. Правосторонний предел рассматривается аналогично. При достаточно малом $|\Delta|, \tau_{1}, \ldots, \tau_{k} \subseteq[0 ; 1]$,

$$
\begin{aligned}
& \frac{1}{\Delta}(h(t+\Delta)-h(t))\left(\tau_{1}, \ldots, \tau_{k}\right) \\
& \quad=\frac{1}{\Delta} \int_{\tau_{i}}^{t} \sum_{i=1}^{k} f^{\prime}(r) d r \mathbb{I}_{[t+\Delta ; t]}\left(\tau_{i}\right) \mathbb{I}_{\left[0 ; \tau_{i}\right]}^{\otimes k-1}\left(\tau_{1}, \ldots, \tau_{i-1}, \tau_{i+1}, \ldots, \tau_{k}\right) \\
& \quad+\frac{1}{\Delta} \sum_{i=1}^{k} \int_{t+\Delta}^{t} f^{\prime}(r) d r \mathbb{I}_{[0 ; t+\Delta]}\left(\tau_{i}\right) \mathbb{I}_{\left[0 ; \tau_{i}\right]}^{\otimes k-1}\left(\tau_{1}, \ldots, \tau_{i-1}, \tau_{i+1}, \ldots, \tau_{k}\right) .
\end{aligned}
$$

Найдем $L_{2}$-норму первого слагаемого:

$$
\begin{aligned}
& \frac{1}{\Delta^{2}} \int_{0}^{1} \ldots \int_{0}^{1}\left(\sum_{i=1}^{k} \int_{\tau_{i}}^{t} f^{\prime}(r) d r \mathbb{I}_{[t+\Delta ; t]}\left(\tau_{i}\right)\right. \\
& \left.\quad \times \mathbb{I}_{\left[0 ; \tau_{i}\right]}^{\otimes k-1}\left(\tau_{1}, \ldots, \tau_{i-1}, \tau_{i+1}, \ldots, \tau_{k}\right)\right)^{2} d \tau_{1} \cdots d \tau_{k} \\
& =\frac{1}{\Delta^{2}} k \int_{t+\Delta}^{t} \tau^{k-1}\left(\int_{\tau}^{t} f^{\prime}(r) d r\right)^{2} d \tau \leqslant \frac{1}{\Delta^{2}} k C^{2} \int_{t+\Delta}^{t} \tau^{k-1}(t-\tau)^{2} d \tau \\
& \leqslant \frac{1}{\Delta^{2}} k C^{2} \int_{t+\Delta}^{t} \tau^{k-1}(t-\tau)^{2} d \tau \leqslant k C^{2}|\Delta| \frac{1}{3} \rightarrow 0, \quad \Delta \rightarrow-0 .
\end{aligned}
$$

Здесь $C=\max \left\{\left|f^{\prime}(r)\right|: r \in[0 ; 1]\right\}$.

Аналогично проверяется, что второе слагаемое сходится в $L_{2}$-норMe $\mathrm{K}$

$$
\sum_{i=1}^{k} f^{\prime}(t) \mathbb{I}_{[0 ; t]}\left(\tau_{i}\right) \mathbb{I}_{\left[0 ; \tau_{i}\right]}^{\otimes k-1}\left(\tau_{1}, \ldots, \tau_{i-1}, \tau_{i+1}, \ldots, \tau_{k}\right)=f^{\prime}(t) \mathbb{1}_{[0 ; t]}^{\otimes k}\left(\tau_{1}, \ldots, \tau_{k}\right)
$$

Лемма доказана.

Для того чтобы воспользоваться леммой 4.1 при определении производных для ядер из разложения Ито-Винера для $G_{\varepsilon}^{s, t}(x)$, отметим еще 
следующие простые соотношения:

$$
\begin{aligned}
& \int_{\mathbf{R}}(x+u)_{+} H_{k}\left(\frac{u}{\sqrt{\tau}}\right) p_{\tau}(u) d u=\int_{\mathbf{R}}(x+v \sqrt{\tau})_{+} H_{k}(v) p_{1}(v) d v, \\
& \int_{\mathbf{R}} \mathbb{I}_{[0 ;+\infty]}(x+u) H_{k}\left(\frac{u}{\sqrt{\tau}}\right) p_{\tau}(u) d u \\
& \quad=\int_{\mathbf{R}} \mathbb{I}_{[0 ;+\infty)}(x+v \sqrt{\tau}) H_{k}(v) p_{1}(v) d v, \\
& H_{k}(v)=v H_{k-1}(v)-H_{k-1}^{\prime}(v), \quad k \geqslant 1, \\
& \frac{d}{d t} \int_{\mathbf{R}}(x+v \sqrt{t})_{+} H_{k}(v) p_{1}(v) d v \\
& \quad=\frac{1}{2 \sqrt{t}} \int_{\mathbf{R}} \mathbb{I}_{[0 ;+\infty)}(x+v \sqrt{t}) v H_{k}(v) p_{1}(v) d v, \\
& \frac{d}{d t} \int_{\mathbf{R}} \mathbb{I}_{[0 ;+\infty)}(x+v \sqrt{t}) H_{k}(v) p_{1}(v) d v=\frac{1}{2 \sqrt{t}} \frac{x}{\sqrt{t}} H_{k}\left(\frac{x}{\sqrt{t}}\right) p_{t}(x),
\end{aligned}
$$

$t \in(0 ; 1]$. Теперь, с учетом приведенных равенств, получаем производные по $t$ для ядер из разложения Ито-Винера для $G_{\varepsilon}^{s, t}(x)$. При $k=0$ имеем

$$
\begin{aligned}
& \frac{d}{d t} \int_{\mathbf{R}}(x+u)_{+} p_{t-s+\varepsilon}(u) d u \\
& \quad=\frac{1}{2 \sqrt{t-s+\varepsilon}} \int_{\mathbf{R}} \mathbb{I}_{[0 ;+\infty)}(x+v \sqrt{t-s+\varepsilon}) v p_{1}(v) d v \\
& \quad=\frac{1}{2} \frac{1}{\sqrt{t-s+\varepsilon}} \int_{\mathbf{R}} \mathbb{I}_{[0 ;+\infty)}(x+u) \frac{u}{\sqrt{t-s+\varepsilon}} p_{t-s+\varepsilon}(u) d u=\frac{1}{2} p_{t-s+\varepsilon}(x) .
\end{aligned}
$$

Рассмотрим случай $k \geqslant 1$ с учетом равенства (4.5). Имеем

$$
\begin{aligned}
\frac{d}{d t}\{ & {\left[\frac{2}{k !} \frac{1}{(\sqrt{t-s+\varepsilon})^{k}} \mathbb{I}_{[s-\varepsilon ; t]}^{\otimes k} \int_{\mathbf{R}}(x+u)_{+} H_{k}\left(\frac{u}{\sqrt{t-s+\varepsilon}}\right) p_{t-s+\varepsilon}(u) d u\right.} \\
& \left.-\frac{2}{k !} \frac{1}{\sqrt{\varepsilon}} \mathbb{I}_{[s-\varepsilon ; s]}^{\otimes k} \int_{\mathbf{R}}(x+u)_{+} H_{k}\left(\frac{u}{\sqrt{\varepsilon}}\right) p_{\varepsilon}(u) d u\right] \\
& -\frac{2}{k !} \frac{1}{\left(\sqrt{r^{*}-s+\varepsilon}\right)^{k-1}} \mathbb{I}_{\left\{r^{*} \in[s ; t]\right\}} \mathbb{I}_{\left\{r_{*} \in[s-\varepsilon ; t]\right\}} \\
& \left.\times \int_{\mathbf{R}} \mathbb{I}_{[0 ;+\infty]}(x+u) H_{k-1}\left(\frac{u}{\sqrt{r^{*}-s+\varepsilon}}\right) p_{r^{*}-s+\varepsilon}(u) d u\right\} \\
= & \frac{d}{d t}\left\{\frac{2}{k !} \frac{1}{(\sqrt{t-s+\varepsilon})^{k}} \mathbb{I}_{[s-\varepsilon ; t]}^{\otimes k} \int_{\mathbf{R}}(x+v \sqrt{t-s+\varepsilon})_{+} H_{k}(v) p_{1}(v) d v\right. \\
& \quad-\frac{2}{k !} \frac{1}{\left(\sqrt{r^{*}-s+\varepsilon}\right)^{k-1}} \mathbb{I}_{\left\{r^{*} \in[s ; t]\right\}} \mathbb{I}_{\left\{r_{*} \in[s-\varepsilon ; t]\right\}} \\
& \left.\times \int_{\mathbf{R}} \mathbb{I}_{[0 ;+\infty)}\left(x+v \sqrt{r^{*}-s+\varepsilon}\right) H_{k-1}(v) p_{1}(v) d v\right\}
\end{aligned}
$$




$$
\begin{aligned}
& =\frac{d}{d t}\left\{\frac{2}{k !} \frac{1}{(\sqrt{t-s+\varepsilon})^{k-1}} \mathbb{I}_{[s-\varepsilon ; t]}^{\otimes k}\right. \\
& \times \int_{\mathbf{R}} \mathbb{I}_{[0 ;+\infty)}(x+v \sqrt{t-s+\varepsilon}) H_{k-1}(v) p_{1}(v) d v \\
& -\frac{2}{k !} \frac{1}{\left(\sqrt{r^{*}-s+\varepsilon}\right)^{k-1}} \mathbb{I}_{[s-\varepsilon ; t]}^{\otimes k} \\
& \left.\times \int_{\mathbf{R}} \mathbb{I}_{[0 ;+\infty)}\left(x+v \sqrt{r^{*}-s+\varepsilon}\right) H_{k-1}(v) p_{1}(v) d v\right\} \\
& =\frac{2}{k !} \mathbb{H}_{[s-\varepsilon ; t]}^{\otimes k} \frac{d}{d t}\left(\frac{1}{(\sqrt{t-s+\varepsilon})^{k-1}}\right. \\
& \left.\times \int_{\mathbf{R}} \mathbb{1}_{[0 ;+\infty)}(x+v \sqrt{t-s+\varepsilon}) H_{k-1}(v) p_{1}(v) d v\right) \\
& =\frac{2}{k !} \mathbb{I}_{[s-\varepsilon ; t]}^{\otimes k}\left\{\frac{1}{2(\sqrt{t-s+\varepsilon})^{k}} \frac{x}{\sqrt{x-s+\varepsilon}} H_{k-1}\left(\frac{x}{\sqrt{t-s+\varepsilon}}\right) p_{t-s+\varepsilon}(x)\right. \\
& \left.+\frac{k-1}{2} \frac{1}{(\sqrt{t-s+\varepsilon})^{k+1}} \int_{\mathbf{R}} \mathbb{I}_{[0 ;+\infty)}(x+v \sqrt{t-s+\varepsilon}) H_{k-1}(v) p_{1}(v) d v\right\} \\
& =\frac{2}{k !} \mathbb{I}_{[s-\varepsilon ; t]}^{\otimes k}\left\{\frac{1}{2(\sqrt{t-s+\varepsilon})^{k}} \frac{x}{\sqrt{t-s+\varepsilon}} H_{k}\left(\frac{x}{\sqrt{t-s+\varepsilon}}\right) p_{t-s+\varepsilon}(x)\right. \\
& \left.-\frac{k-1}{2} \frac{1}{(\sqrt{t-s+\varepsilon})^{k}} H_{k-2}\left(\frac{x}{\sqrt{t-s+\varepsilon}}\right) p_{t-s+\varepsilon}(x)\right\} \\
& =\frac{1}{k !} \mathbb{I}_{[s-\varepsilon ; t]}^{\otimes k} \frac{1}{(\sqrt{t-s+\varepsilon})^{k}} p_{t-s+\varepsilon}(x) \\
& \times\left\{\frac{x}{\sqrt{t-s+\varepsilon}} H_{k-1}\left(\frac{x}{\sqrt{t-s+\varepsilon}}\right)-(k-1) H_{k-2}\left(\frac{x}{\sqrt{t-s+\varepsilon}}\right)\right\} \\
& =\frac{1}{k !} \mathbb{I}_{[s-\varepsilon ; t]}^{\otimes k} \frac{1}{(\sqrt{t-s+\varepsilon})^{k}} H_{k}\left(\frac{x}{\sqrt{t-s+\varepsilon}}\right) p_{t-s+\varepsilon}(x) \text {. }
\end{aligned}
$$

Следовательно, с учетом уже встречавшейся оценки для многочленов Эрмита, $G_{\varepsilon}^{s, t}(x)$ имеет непрерывную производную по $t$ в пространстве $W_{-1}^{0,1}$ и эта производная задает обобщенное случайное отображение порядка 1. Для того чтобы воспользоваться теоремой 4.1 , рассмотрим нулевой член разложения Ито-Винера производной: $\alpha_{0}(x, \varepsilon, s, t)=p_{t-s+\varepsilon}(x)$. Для всякой $\varphi \in S^{\prime}$ сейчас

$$
\left|\int_{\mathbf{R}} \alpha_{0}(x, \varepsilon, s, t) \varphi(x) d x\right|=\left|\int_{\mathbf{R}} p_{t-s+\varepsilon} \varphi(x) d x\right| \leqslant \max _{\mathbf{R}}|\varphi(x)|=\|\varphi\|_{0} .
$$

Следовательно, наш функционал $G$ задается сейчас обобщенной функцией нулевого порядка, которая, как и следовало ожидать, является $\delta$ функцией, сосредоточенной в точке 0 :

$$
\forall \varphi \in S: \quad \lim _{t-s+\varepsilon \rightarrow 0} \int_{\mathbf{R}} \varphi(x) \alpha_{0}(x, \varepsilon, s, t) d x=\varphi(0) \text {. }
$$


Приведенные вычисления могут быть без труда перенесены на случай, когда рассматривается аддитивный однородный функционал $J$ от $w$, соответствующий конечной мере $\mu[1]$. В этом случае $J$ оказывается обобщенным однородным функционалом в смысле определения 2.4, порожденным конечной мерой $\mu$, которая рассматривается как обобщенная функция порядка 0 на пространстве $S$.

3 а м е ч а н и е. В статье рассматривался случай одномерного винеровского процесса, однако вышеизложенные рассуждения нетрудно адаптировать для случая размерности, большей единицы.

\section{СПИСОК ЛИТЕРАТУРЫ}

1. Дынжин Е. Б. Марковские процессы. М.: Физматгиз, 1963, 860 с.

2. He S.W., Yang W.Q., Yao R.Q., Wang J. G. Local times of self-intersection for multidimensional Brownian motion. - Nagoya Math. J., 1995, v. 138, p. 51-64.

3. Imkeller P., Perez-Abreu V., Vives J. Chaos expansions of double intersection local time of Brownian motion in $\mathbf{R}^{d}$ and renormalization. - Stochastic Process. Appl., 1995 , v. 56 , № 1 , p. $1-34$.

4. Watanabe $H$. The local time of self-intersections of Brownian motions as generalized Brownian functionals. - Letters in Math. Phys., 1991, v. 23, № 1, p. 1-9.

5. Watanabe $S$. Lectures on Stochastic Differential Equations and Malliavin Calculus. Berlin-Heidelberg: Springer-Verlag, 1984, $118 \mathrm{p}$.

6. Hida T. Analysis of Brownian Functionals. Carleton Mathematical Lecture Notes, № 13. Ottawa: Carleton Univ., 1975, 61 p.

7. Дороговчев $A . A$. Стохастический анализ и случайные отображения в гильбертовом пространстве. Киев: Наукова думка, 1992, 120 с.

8. Скороход А.В. Случайные линейные операторы. Киев: Наукова думка, 1979, $200 \mathrm{c}$.

9. Дороговчев A.A. О суперпозиции случайных отображений в гильбертовом пространстве. - Теория вероятн. и ее примен., 1989, т. 34, в. 2, с. 124-130.

10. Сеге Г. Ортогональные многочлены. М.: ИЛ, 1962,500 с.

11. Ватанабэ C., Икэда $H$. Стохастические дифференциальные уравнения и диффузионные процессы. М.: Наука, 1986, 448 с.

12. Бакун В. В. Об обобщенном локальном времени для процесса броуновского движения. - Укр. матем. журн., 2000, т. 52, № 2, с. 157-164.

Поступила в редакцию

20.IV. 2000 